\title{
Time-Related Changes in Relationships Between the Keys Score, Dietary Lipids, and Serum Total Cholesterol in Japan - NIPPON DATA80/90/2010 -
}

\author{
Yukiko Okami, PhD; Hirotsugu Ueshima, MD; Yasuyuki Nakamura, MD; Keiko Kondo, PhD; \\ Aya Kadota, MD; Nagako Okuda, MD; Tomonori Okamura, MD; Katsuyuki Miura, MD \\ for the NIPPON DATA80/90 and NIPPON DATA2010 Research Groups
}

\begin{abstract}
Background: In recent years, individuals with high serum total cholesterol (TC) have been more likely to modify their diet by reducing their intake of dietary lipids. The aim of this study was to investigate time-related changes in the relationships between the Keys score, dietary lipids, and TC between 1980 and 2010 in representative Japanese populations.
\end{abstract}

Methods and Results: We used 3 cross-sectional cohort studies conducted among Japanese representative populations in 1980, 1990, and 2010 (NIPPON DATA80/90/2010) for the analyses. We analyzed data for 10,365, 7,714, and 2,657 participants, respectively, in each dataset. The relationships between TC and the Keys score (calculated by dietary lipids, cholesterol, and energy intake)/ dietary lipids were examined. In men, a 1-unit higher Keys score correlated with $0.92 \mathrm{mg} / \mathrm{dL}$ and $0.64 \mathrm{mg} / \mathrm{dL}$ TC in 1980 and 1990 , respectively, but not in 2010. In women, a 1 -unit higher Keys score correlated with $0.70 \mathrm{mg} / \mathrm{dL}$ and $0.74 \mathrm{mg} / \mathrm{dL}$ TC in 1980 and 1990 , respectively, but with $0.33 \mathrm{mg} / \mathrm{dL}$ in 2010. In men and women, dietary cholesterol, saturated fatty acid (SFA), and trans fatty acid (TFA) levels were positively associated with TC concentrations in 1980 and 1990, but not in 2010.

Conclusions: In these representative Japanese populations, a higher Keys score as well as dietary cholesterol, SFA, and TFA levels correlated with higher TC concentrations in 1980/1990, but the relationships were weaker or not observed in 2010.

Key Words: Cohort study; Dietary cholesterol; Dietary lipids; Keys score; Serum total cholesterol

$\mathbf{J}$ apan achieved a remarkable breakthrough in the improvement of hypercholesterolemia in the late 20th century: the introduction of statin drugs in 1989:1 the dissemination of dietary modifications and restrictions on dietary cholesterol and lipids in the diet through the growth of mass media and in the clinical setting since the late $1960 \mathrm{~s}^{\mathbf{2}, 3}$ the enactment of the Industrial Safety and Health Law that mandated annual health check-ups for employees in 1972; and the enactment of the Law for the Welfare of the Elderly in $1983 .{ }^{4}$ Under the health check-up system, detection of hypercholesterolemia and subsequent lifestyle modifications became available, particularly for middleaged and elderly individuals.

In the context of these developments, we hypothesized that individuals with higher serum total cholesterol (TC) levels in recent years would be more likely to modify their diets (reduce dietary lipids and cholesterol intake) and take lipid-lowering medications than those with lower TC concentrations; if a sufficient number of individuals do this, dietary lipids will not or will be inversely related to TC. Although previous studies reported that individuals with hypercholesterolemia modify their diets and restrict their intake of dietary lipids, ${ }^{\mathbf{5 , 6}}$ time-related changes in the relationship between dietary lipids and TC against this background have not yet been examined.

Therefore, we examined changes in the relationships between the Keys score (dietary lipids) and TC between 1980 and 2010 in representative Japanese populations using NIPPON DATA80/90/2010.

\section{Methods}

\section{Study Design and Participants}

A pooled dataset of baseline data for analysis was obtained from the National Integrated Project for the Prospective Observation of Non-communicable Diseases and its Trends

Received May 29, 2018; revised manuscript received September 8, 2018; accepted September 26, 2018; J-STAGE Advance Publication released online October 18, 2018 Time for primary review: 28 days

Center for Epidemiologic Research in Asia (Y.O., H.U., A.K., K.M.), Department of Public Health (H.U., Y.N., K.K., A.K., N.O., T.O., K.M.), Shiga University of Medical Science, Otsu; Department of Food Science and Human Nutrition, Faculty of Agriculture, Ryukoku University, Kyoto (Y.N.); Department of Health and Nutrition, University of Human Arts and Sciences, Saitama (N.O.); and Department of Preventive Medicine and Public Health, School of Medicine, Keio University, Tokyo (T.O.), Japan

Mailing address: Yukiko Okami, PhD, Center for Epidemiological Research in Asia, Shiga University of Medical Science, Seta Tsukinowa-cho, Otsu 520-2192, Japan. E-mail: okami@belle.shiga-med.ac.jp

ISSN-1346-9843 All rights are reserved to the Japanese Circulation Society. For permissions, please e-mail: cj@j-circ.or.jp 
in the Aged conducted in 1980, 1990, and 2010 (NIPPON DATA80/90/2010). The methods of these studies are described in detail elsewhere. ${ }^{7,8}$

NIPPON DATA80/90 was the combined data of participants in the National Survey on Circulatory Disorders (NSCD) and the dietary estimates from the National Nutrition Survey conducted in 1980 and 1990, respectively. Participants were those persons surveyed for the $3 \mathrm{rd}$ and 4th NSCD, conducted in 1980 and 1990. They were residents of 300 survey districts throughout Japan, randomly selected for the National Surveys by the Ministry of Health, Labour and Welfare of Japan. The participants for NIPPON DATA2010 were those who agreed to participate in the baseline survey and signed the consent form among participants in 300 districts throughout Japan, randomly selected for the NHNS by the Ministry of Health, Labour and Welfare of Japan in 2010. Surveys were conducted in a local health center, and consisted of physical examination, blood tests, and a self-administered questionnaire in which participants were asked about their habits of alcohol drinking, smoking, treatment for hypercholesterolemia (1990 and 2010), and history of cardiovascular disease (CVD). In NIPPON DATA90/2010, participants were asked about "...controlling your diet (type of food, meal size, amount of fat, or balance between meat and fish". If they answered "yes" to at least one of the above in the questionnaire, they were considered to be controlling their diet. Height in stocking feet and weight in light clothing were measured. Body mass index (BMI) was calculated as weight (in $\mathrm{kg}$ ) divided by the square of height (in $\mathrm{m}$ ).

The total numbers of community dwellers aged 30 years (20 years for NIPPON DATA2010) and older who were eligible and invited to participate were 10,546 (4,639 men and 5,907 women) in 1980, 8,383 (3,504 men and 4,879 women) in 1990, and 2,891 (1,236 men and 1,655 women) in 2010. People with an implausible energy intake $(<500$ or $>5,000 \mathrm{kcal} /$ day) or missing data for age, BMI, drinking, smoking, TC, and Keys score (dietary lipids), or who were under 30 years old (for NIPPON DATA2010) were excluded from the analysis, leaving 10,365 (4,558 men and 5,807 women) for NIPPON DATA80, 7,714 (3,220 men and 4,494 women) for NIPPON DATA90, and 2,657 (1,130 men and 1,527 women) for NIPPON DATA2010 (Figure S1). The Institutional Review Board of Shiga University of Medical Science (No. 22-29, 2010) approved this study.

\section{Dietary Assessment}

Dietary intakes for NIPPON DATA80/90 were assessed for each household using a 3-day semi-weighing dietary record method. Trained dieticians reviewed dietary records with the participants and edited them as necessary. Survey data were processed centrally to calculate total energy intake and the intake of nutrients and food groups. In this study, the nutrient intake of each household member was estimated by proportional distribution method, dividing the household intake data of the National Nutrition Survey in 1980/1990 proportionally with average intakes, as categorized by sex and age groups. In addition, other nutrients known to have effects on CVD risk factors, such as trans fatty acids (TFA), were calculated based on the INTERMAP Food Table and INTERMAP Japan dietary survey data. ${ }^{7}$ The detailed procedure and results of the validation for these estimations have been reported elsewhere. ${ }^{7,9}$ In NIPPON DATA2010, 1-day dietary surveys with semi-weighing household dietary records and information on the food distribution ratio among family members were conducted by trained dietary interviewers. ${ }^{8}$

Dietary intakes of cholesterol $(\mathrm{mg} / 1,000 \mathrm{kcal})$, saturated fatty acids (SFA) (\%kcal), polyunsaturated fatty acids (PUFA) (\%kcal), monounsaturated fatty acids (MUFA) (\%kcal), TFA ( $\% \mathrm{kcal})$, and dietary fiber $(\mathrm{g} / 1,000 \mathrm{kcal})$ were used in the analysis. The Keys score, which has been widely used as a reflector of TC in previous studies, ${ }^{10-12}$ was calculated using the following equation: ${ }^{13} \varphi$ (unit)= $2.7 \times \mathrm{S}-1.35 \times \mathrm{P}+1.5 \times \mathrm{C}^{1 / 2}$, where $\mathrm{S}$ is SFA (\%kcal), $\mathrm{P}$ is PUFA (\%kcal), and C is dietary cholesterol ( $\mathrm{mg} / 1,000 \mathrm{kcal})$. Keys et al developed this equation to predict the average change in the serum cholesterol level by dietary change. One score increment in the Keys score is expected to correspond to an increase in serum cholesterol by $1 \mathrm{mg} / \mathrm{dL}$. The predicted change in serum cholesterol from the Keys equation is highly correlated with the observed change in serum cholesterol $(\mathrm{r}=0.92) .{ }^{13}$

\section{Serum Lipid Measurements}

Non-fasting blood samples were obtained at each baseline for NIPPON DATA80/90/2010. Serum was separated and centrifuged soon after blood coagulation. Samples were shipped to one laboratory for blood chemistry measurements. TC was measured enzymatically and standardized by the Center for Disease Control and Prevention/National Heart, Lung, and Blood Institute (CDC-NHLBI) Lipids Standardization Program and US Cholesterol Reference Method Laboratory Network (CRMLN). ${ }^{14,15}$ Serum high-density lipoprotein (HDL) cholesterol was measured in 1990 and 2010, but not in 1980 .

\section{Statistical Analysis}

In the description of the characteristics of participants for variables related to $\mathrm{TC}(\mathrm{mg} / \mathrm{dL})$, means and standard deviations (SD) or percentages were calculated separated by sex and datasets, with an analysis of variance (ANOVA) test or chi-squared test for comparisons among the 3 datasets (NIPPON DATA 80/90/2010). The relationship between the Keys score and TC $(<200,200-239,>239)$ was compared among the 3 datasets with the ANOVA test. A multiple linear regression analysis was used to examine the relationship between TC as a dependent variable and the Keys score as an independent variable and required covariates, including age, BMI, smoking status, drinking status, and dietary fiber intake. Dummy variables were the smoking status (non-smoker, ex-smoker, current smoker) and drinking status (non-drinker, ex-drinker, current drinker). Model 1 was adjusted for age, while Model 2 was further adjusted for BMI, smoking status, drinking status, and dietary fiber. We then calculated the difference in TC $(\mathrm{mg} / \mathrm{dL})$ per 1-unit increase in the Keys score. The same analyses were conducted among the participants excluded for being treated for hypercholesterolemia and controlling their diet (1990 and 2010) or with a history of CVD. As a subgroup analysis, we stratified those controlling their diet in Model 2 in order to assess the effects on the relationship between the Keys score and TC. Stratification by age at 50 or 55 years old or by age group (30-39, 40-59, 60-79, and 80-95 years old) was also performed in order to observe the effects of treatment, diet, and differences between age groups. Another multiple regression analysis was conducted between $\mathrm{TC}$ as a dependent variable and $1 \mathrm{SD}$ of an increase in dietary cholesterol $(\mathrm{mg} / 1,000 \mathrm{kcal}), \mathrm{SFA}(\% \mathrm{kcal})$, 


\begin{tabular}{|c|c|c|c|c|}
\hline Men & $\begin{array}{c}1980 \\
(n=4,558)\end{array}$ & $\begin{array}{c}1990 \\
(n=3,220)\end{array}$ & $\begin{array}{c}2010 \\
(n=1,130)\end{array}$ & $P$ value \\
\hline Age (years) & $49.9 \pm 13.4$ & $53.3 \pm 13.6$ & $61.5 \pm 13.9$ & $<0.001$ \\
\hline $\mathrm{BMI}\left(\mathrm{kg} / \mathrm{m}^{2}\right)$ & $22.5 \pm 2.9$ & $23.0 \pm 3.0$ & $24.0 \pm 3.2$ & $<0.001$ \\
\hline Smoking status, $\mathrm{n}(\%)$ & & & & $<0.001$ \\
\hline Non-smoker & $830(18.2)$ & $657(20.4)$ & $308(27.3)$ & \\
\hline Ex-smoker & $847(18.6)$ & $779(24.2)$ & $501(44.3)$ & \\
\hline Current smoker & $2,881(63.2)$ & $1,784(55.4)$ & $321(28.4)$ & \\
\hline Drinking status, $\mathrm{n}(\%)$ & & & & $<0.001$ \\
\hline Non-drinker & $2,127(46.7)$ & $1,126(35.0)$ & $396(35.0)$ & \\
\hline Ex-drinker & $263(5.8)$ & $226(7.0)$ & $36(3.2)$ & \\
\hline Current drinker & $2,168(47.6)$ & $1,868(58.0)$ & $698(61.8)$ & \\
\hline Dietary energy (kcal/day) & $2,399 \pm 482$ & $2,323 \pm 457$ & $2,145 \pm 557$ & $<0.001$ \\
\hline Dietary fat (\%kcal) & $20.0 \pm 5.2$ & $22.3 \pm 4.5$ & $23.2 \pm 6.7$ & $<0.001$ \\
\hline Dietary cholesterol (mg/1,000 kcal) & $164.6 \pm 55.2$ & $183.1 \pm 53.0$ & $155.7 \pm 83.0$ & $<0.001$ \\
\hline SFA (\%kcal) & $5.65 \pm 1.47$ & $5.90 \pm 1.35$ & $6.04 \pm 2.32$ & $<0.001$ \\
\hline PUFA (\%kcal) & $5.27 \pm 1.39$ & $5.58 \pm 1.30$ & $5.31 \pm 1.87$ & $<0.001$ \\
\hline MUFA (\%kcal) & $7.44 \pm 1.95$ & $7.90 \pm 1.76$ & $7.84 \pm 2.79$ & $<0.001$ \\
\hline TFA (\%kcal) & $0.32 \pm 0.19$ & $0.33 \pm 0.17$ & - & $0.019^{*}$ \\
\hline Total fiber ( $\mathrm{g} / 1,000 \mathrm{kcal})$ & $7.7 \pm 1.8$ & $7.0 \pm 1.9$ & $7.8 \pm 3.0$ & $<0.001$ \\
\hline Keys score (unit) ${ }^{\star \star}$ & $27.1 \pm 5.7$ & $28.5 \pm 5.1$ & $27.1 \pm 8.5$ & $<0.001$ \\
\hline Serum total cholesterol $(\mathrm{mg} / \mathrm{dL})^{\star * *}$ & $186.4 \pm 32.8$ & $198.6 \pm 36.8$ & $202.2 \pm 34.1$ & $<0.001$ \\
\hline Non-HDL-cholesterol (mg/dL) & - & $148.3 \pm 38.7$ & $144.9 \pm 34.3$ & $<0.001^{*}$ \\
\hline Being treated for hypercholesterolemia, $\mathrm{n}(\%)$ & - & $216(6.7)$ & $163(14.4)$ & $<0.001$ \\
\hline Controlling their diet, $\mathrm{n}(\%)$ & - & $1,891(58.7)$ & $776(68.7)$ & $<0.001$ \\
\hline CVD history, $\mathrm{n}(\%)$ & $144(3.2)$ & $170(5.3)$ & $132(11.7)$ & $<0.001$ \\
\hline Women & $(n=5,807)$ & $(n=4,494)$ & $(n=1,527)$ & \\
\hline Age (years) & $50.0 \pm 13.5$ & $52.4 \pm 13.9$ & $59.3 \pm 14.7$ & $<0.001$ \\
\hline $\mathrm{BMI}\left(\mathrm{kg} / \mathrm{m}^{2}\right)$ & $22.8 \pm 3.4$ & $22.9 \pm 3.3$ & $22.8 \pm 3.5$ & 0.619 \\
\hline Smoking status, $\mathrm{n}(\%)$ & & & & $<0.001$ \\
\hline Non-smoker & $5,097(87.8)$ & $3,955(88.0)$ & $1,291(84.5)$ & \\
\hline Ex-smoker & $139(2.4)$ & $117(2.6)$ & $135(8.8)$ & \\
\hline Current smoker & $571(9.8)$ & $422(9.4)$ & $101(6.6)$ & \\
\hline Drinking status, $n(\%)$ & & & & $<0.001$ \\
\hline Non-drinker & $5,538(95.4)$ & $4,149(92.3)$ & $1,158(75.8)$ & \\
\hline Ex-drinker & $98(1.7)$ & $48(1.1)$ & $21(1.4)$ & \\
\hline Current drinker & $171(2.9)$ & $297(6.6)$ & $348(22.8)$ & \\
\hline Dietary energy (kcal/day) & $1,929 \pm 390$ & $1,865 \pm 365$ & $1,753 \pm 431$ & $<0.001$ \\
\hline Dietary fat (\%kcal) & $21.7 \pm 5.7$ & $24.4 \pm 5.0$ & $25.7 \pm 7.1$ & $<0.001$ \\
\hline Dietary cholesterol (mg/1,000 kcal) & $178.6 \pm 59.0$ & $199.8 \pm 59.0$ & $170.6 \pm 90.1$ & $<0.001$ \\
\hline SFA (\%kcal) & $6.18 \pm 1.66$ & $6.48 \pm 1.54$ & $6.89 \pm 2.57$ & $<0.001$ \\
\hline PUFA (\%kcal) & $5.76 \pm 1.52$ & $6.08 \pm 1.40$ & $5.73 \pm 2.03$ & $<0.001$ \\
\hline MUFA (\%kcal) & $8.12 \pm 2.16$ & $8.66 \pm 1.97$ & $8.57 \pm 3.00$ & $<0.001$ \\
\hline TFA (\%kcal) & $0.35 \pm 0.22$ & $0.37 \pm 0.19$ & - & $0.005^{\star}$ \\
\hline Fiber (g/1,000 kcal) & $9.2 \pm 2.1$ & $8.4 \pm 2.3$ & $9.2 \pm 3.5$ & $<0.001$ \\
\hline Keys score (unit) ${ }^{\star \star}$ & $28.7 \pm 6.1$ & $30.3 \pm 5.7$ & $29.7 \pm 9.3$ & $<0.001$ \\
\hline Serum total cholesterol $(\mathrm{mg} / \mathrm{dL})^{\star \star *}$ & $191.2 \pm 34.0$ & $206.9 \pm 38.8$ & $210.0 \pm 35.7$ & $<0.001$ \\
\hline Non-HDL-cholesterol (mg/dL) & - & $150.0 \pm 40.2$ & $142.4 \pm 36.7$ & $<0.001^{*}$ \\
\hline Being treated for hypercholesterolemia, $n(\%)$ & - & $352(7.8)$ & $271(17.8)$ & $<0.001$ \\
\hline Controlling their diet, $\mathrm{n}(\%)$ & - & $3,079(68.5)$ & $1,170(76.6)$ & $<0.001$ \\
\hline CVD history, $\mathrm{n}(\%)$ & $130(2.2)$ & $170(3.8)$ & $80(5.2)$ & $<0.001$ \\
\hline
\end{tabular}

Values are mean $\pm \mathrm{SD}$, or $\mathrm{n}(\%)$ (as indicated). $\mathrm{P}$ values were obtained using ANOVA or the chi-squared test. *Unpaired $\mathrm{t}$-test. ${ }^{* *}$ Keys score $=2.7 \times S-1.35 \times P+1.5 \times \sqrt{C}$. S, saturated fatty acids (\%kcal); $P$, polyunsaturated fatty acids (\%kcal); C, dietary cholesterol (mg/1,000 kcal). ${ }^{* \star *}$ SI conversion factors: In order to convert serum total cholesterol to $\mathrm{mmol} / \mathrm{L}$, multiply values by 0.0259 . BMI, body mass index; CVD, cardiovascular disease; HDL, high-density lipoprotein; MUFA, monounsaturated fatty acids; PUFA, polyunsaturated fatty acids; SFA, saturated fatty acids; TFA, trans fatty acids. 


\begin{tabular}{|c|c|c|c|c|c|c|}
\hline & \multicolumn{3}{|c|}{ Men } & \multicolumn{3}{|c|}{ Women } \\
\hline & 1980 & 1990 & 2010 & 1980 & 1990 & 2010 \\
\hline Unadjusted & $(n=4,558)$ & $(n=3,220)$ & $(n=1,130)$ & $(n=5,807)$ & $(n=4,494)$ & $(n=1,527)$ \\
\hline$\beta^{\star \star}$ & 1.00 & 0.74 & 0.00 & 0.06 & -0.08 & 0.18 \\
\hline$(95 \% \mathrm{Cl})$ & $(0.84,1.17)$ & $(0.49,0.99)$ & $(-0.23,0.24)$ & $(-0.09,0.20)$ & $(-0.28,0.12)$ & $(-0.01,0.37)$ \\
\hline$P$ value & $<0.001$ & $<0.001$ & 0.981 & 0.446 & 0.417 & 0.065 \\
\hline \multicolumn{7}{|l|}{ Model 1} \\
\hline$\beta^{\star \star}$ & 0.97 & 0.67 & -0.06 & 0.65 & 0.64 & 0.33 \\
\hline$(95 \% \mathrm{Cl})$ & $(0.80,1.14)$ & $(0.41,0.92)$ & $(-0.29,0.17)$ & $(0.51,0.79)$ & $(0.44,0.84)$ & $(0.14,0.52)$ \\
\hline$P$ value & $<0.001$ & $<0.001$ & 0.624 & $<0.001$ & $<0.001$ & $<0.001$ \\
\hline \multicolumn{7}{|l|}{ Model 2} \\
\hline$\beta^{\star \star}$ & 0.92 & 0.64 & -0.07 & 0.70 & 0.74 & 0.33 \\
\hline$(95 \% \mathrm{Cl})$ & $(0.75,1.09)$ & $(0.39,0.89)$ & $(-0.30,0.17)$ & $(0.55,0.84)$ & $(0.54,0.94)$ & $(0.13,0.53)$ \\
\hline$P$ value & $<0.001$ & $<0.001$ & 0.564 & $<0.001$ & $<0.001$ & 0.001 \\
\hline \multicolumn{7}{|c|}{ Excluded participants being treated for hypercholesterolemia } \\
\hline Model 2 & & $(n=3,004)$ & $(n=967)$ & & $(n=4,142)$ & $(n=1,256)$ \\
\hline$\beta^{\star \star}$ & - & 0.60 & -0.05 & - & 0.73 & 0.34 \\
\hline$(95 \% \mathrm{Cl})$ & & $(0.35,0.85)$ & $(-0.30,0.20)$ & & $(0.53,0.92)$ & $(0.12,0.56)$ \\
\hline$P$ value & - & $<0.001$ & 0.684 & - & $<0.001$ & 0.003 \\
\hline \multicolumn{7}{|c|}{ Excluded participants being treated for hypercholesterolemia or controlling their diet } \\
\hline Model 2 & & $(n=1,280)$ & $(n=320)$ & & $(n=1,381)$ & $(n=332)$ \\
\hline$\beta^{\star \star}$ & - & 0.62 & -0.18 & - & 0.80 & 0.11 \\
\hline$(95 \% \mathrm{Cl})$ & & $(0.24,0.99)$ & $(-0.62,0.26)$ & & $(0.47,1.13)$ & $(-0.26,0.48)$ \\
\hline$P$ value & - & 0.001 & 0.414 & - & $<0.001$ & 0.561 \\
\hline
\end{tabular}

Model 1 was adjusted for age, and Model 2 was further adjusted for BMI, smoking status (non-smoker, ex-smoker, and current smoker), drinking status (non-drinker, ex-drinker, and current drinker), and dietary fiber $(\mathrm{g} / 1,000 \mathrm{kcal})$. ${ }^{*}$ Keys score $=2.7 \times \mathrm{S}-1.35 \times \mathrm{P}+1.5 \times \sqrt{\mathrm{C}}$. $\mathrm{S}$, saturated fatty acids (\%kcal); P, polyunsaturated fatty acids (\% kcal); C, dietary cholesterol $(\mathrm{mg} / 1,000 \mathrm{kcal}) .{ }^{* *} \beta$ is shown as a natural coefficient of the Keys score. BMI, body mass index; $\mathrm{Cl}$, confidence interval.

PUFA (\%kcal), MUFA (\%kcal), TFA (\%kcal), and dietary fiber ( $\mathrm{g} / 1,000 \mathrm{kcal})$, adjusted for age, BMI, smoking status, and drinking status. We calculated the standardized coefficient values of the Keys score, age, BMI, dietary fiber, smoking status, and drinking status in order to clarify the effective size of each independent variable on TC. In addition, non-HDL-cholesterol was compared between 1990 and 2010 and the associations between the Keys score and non-HDL-cholesterol were assessed with linear regression models. All analyses were performed using SAS version 9.4 (SAS Institute, Cary, NC, USA); two-tailed $\mathrm{P}<0.05$ was considered to be significant.

\section{Results}

Participant characteristics in NIPPON DATA80/90/2010 are shown in Table 1. Among men and women, age, current drinkers, dietary fat intake, SFA, TFA, TC, participants being treated for hypercholesterolemia, controlling their diet, and with a CVD history significantly increased, and current smokers and total energy intake decreased between 1980 and 2010. Non-HDL-cholesterol decreased from 1990 to 2010 in both men and women. This was also observed in analyses where participants under treatment for hypercholesterolemia were excluded. Among men, BMI also increased between 1980 and 2010. Dietary intakes of cholesterol and PUFA and the Keys score remained at the same level over time in men and women. The relationship between the Keys score and TC is shown in Table S1. In men, positive correlations were observed in 1980 and 1990 and became negative in 2010. No correlations were found in any dataset for women.

Table 2 shows the results of the linear regression analysis with TC as a dependent variable and the Keys score as an independent variable. In men, a 1-unit higher Keys score was associated with significantly higher TC in 1980, but the positive association was lower in 1990 and absent in 2010. In women, no associations were observed between the Keys score and TC in unadjusted models in any of the datasets. This was consistent with the findings shown in Table S1. However, positive correlations between the Keys score and TC were observed in all datasets after adjustment for age, although the coefficient value was lower and nonsignificant in 2010 (Table 2). Figure S2 shows scatter plots of TC and the Keys score stratified by sex. The attenuation of the relationship between the Keys score and TC from $1980 / 1990$ to 2010 was similarly observed after adjustment for other covariates and among excluded male and female participants being treated for hypercholesterolemia or controlling their diet. Similar results were observed among male and female participants without a history of CVD (data not shown). The association between the Keys score and non-HDL-cholesterol (mg/dL) was also attenuated from 1990 to 2010 (data not shown). Table S2 shows the standardized coefficient values of all independent variables with TC in Model 2. In men, the most associated factor with TC was BMI and second was the Keys score, and both coefficients decreased in 2010. Age was negatively 


\begin{tabular}{|c|c|c|c|c|c|c|}
\hline \multirow{2}{*}{ Keys score } & \multicolumn{3}{|c|}{ Men } & \multicolumn{3}{|c|}{ Women } \\
\hline & 1980 & 1990 & 2010 & 1980 & 1990 & 2010 \\
\hline $30-39$ years old & $(n=1,216)$ & $(n=605)$ & $(n=108)$ & $(n=1,580)$ & $(n=976)$ & $(n=230)$ \\
\hline$\beta^{\star \star}$ & 0.62 & 0.54 & 0.48 & 0.82 & 1.17 & 0.16 \\
\hline$(95 \% \mathrm{Cl})$ & $(0.33,0.91)$ & $(-0.01,1.09)$ & $(-0.48,1.44)$ & $(0.57,1.08)$ & $(0.79,1.55)$ & $(-0.25,0.58)$ \\
\hline$P$ value & $<0.001$ & 0.052 & 0.324 & $<0.001$ & $<0.001$ & 0.441 \\
\hline $40-59$ years old & $(n=2,203)$ & $(n=1,505)$ & $(n=310)$ & $(n=2,773)$ & $(n=2,063)$ & $(n=456)$ \\
\hline$\beta^{\star \star}$ & 1.02 & 0.49 & -0.51 & 0.74 & 0.69 & 0.43 \\
\hline$(95 \% \mathrm{Cl})$ & $(0.76,1.28)$ & $(0.10,0.87)$ & $(-0.94,-0.08)$ & $(0.54,0.94)$ & $(0.39,0.98)$ & $(0.09,0.77)$ \\
\hline$P$ value & $<0.001$ & 0.013 & 0.020 & $<0.001$ & $<0.001$ & 0.014 \\
\hline $60-79$ years old & $(n=1,075)$ & $(n=1,026)$ & $(n=626)$ & $(n=1,350)$ & $(n=1,319)$ & $(n=734)$ \\
\hline$\beta^{\star \star}$ & 1.20 & 0.79 & 0.04 & 0.65 & 0.58 & 0.21 \\
\hline$(95 \% \mathrm{Cl})$ & $(0.86,1.53)$ & $(0.36,1.23)$ & $(-0.27,0.35)$ & $(0.33,0.97)$ & $(0.21,0.96)$ & $(-0.08,0.50)$ \\
\hline$P$ value & $<0.001$ & $<0.001$ & 0.794 & $<0.001$ & 0.002 & 0.162 \\
\hline $80-95$ years old & $(n=64)$ & $(n=84)$ & $(n=86)$ & $(n=104)$ & $(n=136)$ & $(n=107)$ \\
\hline$\beta^{\star *}$ & 0.90 & 1.29 & 0.52 & -0.25 & 1.67 & 0.62 \\
\hline$(95 \% \mathrm{Cl})$ & $(-0.52,2.31)$ & $(-0.02,2.59)$ & $(-0.16,1.20)$ & $(-1.62,1.12)$ & $(0.39,2.94)$ & $(-0.03,1.27)$ \\
\hline$P$ value & 0.211 & 0.054 & 0.130 & 0.714 & 0.011 & 0.063 \\
\hline
\end{tabular}

All models were adjusted for age, BMI, smoking status (non-smoker, ex-smoker, and current smoker), drinking status (non-drinker, ex-drinker and current drinker), and dietary fiber ( $\mathrm{g} / 1,000 \mathrm{kcal})$. ${ }^{*}$ Keys score $=2.7 \times \mathrm{S}-1.35 \times \mathrm{P}+1.5 \times \sqrt{\mathrm{C}}$. S, saturated fatty acids $(\% \mathrm{kcal}) ; \mathrm{P}$, polyunsaturated fatty acids (\%kcal); C, dietary cholesterol $(\mathrm{mg} / 1,000 \mathrm{kcal}) .{ }^{* *} \beta$ is shown as a natural coefficient of the Keys score. $\mathrm{BMI}$, body mass index; $\mathrm{Cl}$, confidence interval.

\begin{tabular}{|c|c|c|c|c|c|c|}
\hline & & Men & & & Women & \\
\hline & $\begin{array}{c}1980 \\
(n=4,558)\end{array}$ & $\begin{array}{c}1990 \\
(n=3,220)\end{array}$ & $\begin{array}{c}2010 \\
(n=1,130)\end{array}$ & $\begin{array}{c}1980 \\
(n=5,807)\end{array}$ & $\begin{array}{c}1990 \\
(n=4,494)\end{array}$ & $\begin{array}{c}2010 \\
(n=1,527)\end{array}$ \\
\hline \multicolumn{7}{|c|}{ Dietary cholesterol } \\
\hline$\beta$ & 1.73 & 1.04 & -0.70 & 1.03 & 1.70 & 0.32 \\
\hline$(95 \% \mathrm{Cl})$ & $(0.50,2.95)$ & $(-0.59,2.67)$ & $(-2.24,0.83)$ & $(0.01,2.06)$ & $(0.42,2.99)$ & $(-0.96,1.60)$ \\
\hline$P$ value & 0.006 & 0.211 & 0.369 & 0.048 & 0.010 & 0.625 \\
\hline \multicolumn{7}{|l|}{ SFA } \\
\hline$\beta$ & 3.70 & 2.51 & -0.44 & 1.63 & 3.43 & 0.16 \\
\hline$(95 \% \mathrm{Cl})$ & $(1.25,6.18)$ & $(-0.74,5.77)$ & $(-2.78,1.89)$ & $(-0.43,3.70)$ & $(0.89,5.98)$ & $(-1.64,1.95)$ \\
\hline$P$ value & 0.003 & 0.130 & 0.710 & 0.120 & 0.008 & 0.862 \\
\hline \multicolumn{7}{|l|}{ PUFA } \\
\hline$\beta$ & -1.36 & 0.07 & -0.12 & -0.46 & 0.37 & -1.78 \\
\hline$(95 \% \mathrm{Cl})$ & $(-3.50,0.78)$ & $(-2.69,2.83)$ & $(-2.22,1.98)$ & $(-2.23,1.30)$ & $(-1.89,2.63)$ & $(-3.49,-0.07)$ \\
\hline$P$ value & 0.214 & 0.960 & 0.912 & 0.606 & 0.751 & 0.042 \\
\hline \multicolumn{7}{|l|}{ MUFA } \\
\hline$\beta$ & 1.80 & 0.45 & 0.94 & 2.21 & 0.21 & 3.15 \\
\hline$(95 \% \mathrm{Cl})$ & $(-1.53,5.12)$ & $(-3.91,4.81)$ & $(-2.07,3.94)$ & $(-0.55,4.97)$ & $(-3.28,3.69)$ & $(0.78,5.52)$ \\
\hline$P$ value & 0.289 & 0.839 & 0.541 & 0.116 & 0.908 & 0.009 \\
\hline \multicolumn{7}{|l|}{ TFA } \\
\hline$\beta$ & 1.50 & 2.11 & - & 2.07 & 1.16 & - \\
\hline$(95 \% \mathrm{Cl})$ & $(0.15,2.85)$ & $(0.13,4.09)$ & & $(0.97,3.16)$ & $(-0.39,2.72)$ & \\
\hline$P$ value & 0.030 & 0.037 & - & $<0.001$ & 0.143 & - \\
\hline \multicolumn{7}{|c|}{ Dietary fiber } \\
\hline$\beta$ & -1.10 & -2.33 & -0.79 & -0.26 & 0.76 & -0.01 \\
\hline$(95 \% \mathrm{Cl})$ & $(-2.45,0.26)$ & $(-4.01,-0.65)$ & $(-2.49,0.92)$ & $(-1.28,0.76)$ & $(-0.47,1.99)$ & $(-1.32,1.30)$ \\
\hline$P$ value & 0.112 & 0.007 & 0.366 & 0.619 & 0.228 & 0.985 \\
\hline
\end{tabular}

Dietary cholesterol, SFA, PUFA, MUFA, TFA, and dietary fiber were included in the same model as independent variables and adjusted for age, BMI, smoking status (non-smoker, ex-smoker, and current smoker), and drinking status (non-drinker, ex-drinker, and current drinker). ${ }^{*} 1$ standard deviation differences for dietary cholesterol, SFA, PUFA, MUFA, TFA, and dietary fiber are shown in Table 1. Abbreviations as in Tables 1,2. 
associated with $\mathrm{TC}$ in men. In women, age was the most strongly associated with TC, BMI was the second, and Keys score was third in all datasets. Furthermore, all coefficient values decreased in 2010 , which was similar to the results obtained for men.

Table 3 shows the results of the linear regression analysis with TC and the Keys score stratified by age groups (3039, 40-59, 60-79, and 80-95 years old) (Model 2). When data were observed horizontally, the positive association between the Keys score and TC abated from 1980/1990 to 2010 in all age groups, except for 80-95-year-old women. In 40-59-year-old men, the positive correlation observed in 1980 became weaker in 1990 and negative in 2010. Women showed smaller changes in coefficient values than men during the time periods examined. The attenuation of relationships between 1980/1990 and 2010 was observed in all stratified groups at 50 or 55 years old $(<50 / \geq 50$ or $<55 / \geq 55$ ) in both men and women (data not shown). When data were observed vertically, the coefficient values in men slightly increased from the younger to older generation in $1980 / 1990$, but not in 2010. In women, coefficient values slightly decreased from the younger to older generation in $1980 / 1990$, but not in 2010 .

Table 4 shows the results of the multiple linear regression analysis with 6 dietary factors as independent variables and TC as a dependent variable. One SD in the dietary intakes of cholesterol, SFA, PUFA, MUFA, TFA, and dietary fiber was included in the same model as independent variables and adjusted for age, BMI, smoking status, and drinking status. When results were observed horizontally, the positive correlation observed for dietary cholesterol and SFA with TC in 1980 was attenuated in 1990 and became negative in 2010 in men. In women, the positive correlation between dietary cholesterol and TC in 1980/1990 became negative in 2010 . TFA positively correlated with TC in men and women. When data were observed vertically, SFA or MUFA had the largest coefficient values in all datasets. The coefficient value of dietary cholesterol on TC was nearly one-half of that of SFA in 1980/1990 for both men and women.

Similar results were obtained in both men and women when excluding participants with a history of CVD, being treated for hypercholesterolemia, and controlling their diet (data not shown).

\section{Discussion}

In men and women, a higher Keys score, dietary cholesterol, and SFA correlated with higher TC concentrations in 1980/1990; however, this relationship was attenuated in 2010. In men, the positive correlation in 1980 was attenuated in 1990 and became negative in 2010. These changes were similar among participants not being treated for hypercholesterolemia or with a history of CVD.

The marked decreases observed in coefficient values for the Keys score, dietary cholesterol, and SFA between 1980/1990 and 2010 suggested frequent restriction of dietary lipid intake among healthy individuals in recent years. Health education on restricting the amount of dietary cholesterol and fat intake in the clinical setting started in the 1960s. 2,3,16 Because of this, and rapid economic growth in Japan, health and diet information was circulated through the mass media. A national survey reported that $37.7 \%$ of individuals in the general population in 1980 were controlling their diet. ${ }^{17}$ The present study indicated that
$50-70 \%$ of individuals in 1990 made a conscious effort to control their diet and this increased to $60-80 \%$ in 2010 . Previous studies reported that "healthism" or "health fads" spread widely throughout the nation from the mid-1970 s. ${ }^{17,18}$ The combined result showed that the proportion of the general population consciously or subconsciously considering their health or diet markedly increased from around the 1980s. Because no correlations were observed between the Keys score and TC in the present study, even among participants not controlling their diet, we assumed that relatively large numbers of participants may unconsciously modify their diet or just did not recognize their attention to their diet. This question needs to be addressed in future studies.

A new nationwide health promotion program started in 2008. This employment-sponsored program, including health screening and subsequent healthcare advice, has improved health awareness in Japan. ${ }^{19}$ The effectiveness of individual education programs for modifying diets and lowering the risk of CVD has been confirmed in a number of studies. ${ }^{\mathbf{1 2 , 2 0 - 2 2}}$ A previous study reported that less educated individuals have higher Keys scores, sodium intake, and blood pressure than more educated individuals because education increases healthy behaviors and lifestyle improvements, specifically in the working generation aged 40-59 years old. ${ }^{\mathbf{1 2} 23}$ The present results support the interpretation that individuals with higher TC abstained from eating dietary lipids significantly more than those with lower TC among men aged 40-59 years old in 2010. Furthermore, the present study adds further information that this paradoxical logic has been frequently observed in recent years, with TC concentrations reflecting dietary lipid intake. Otherwise, participants who knew their TC level was high might have modified their diet intentionally when the survey was conducted. In either case, this phenomenon in which exposure and outcomes are more likely to be opposite (reverse causality or temporal confounding) has already been reported,, $\mathbf{5}$ indicating that individuals with high TC modify their diets and abstain from eating SFA and dietary cholesterol, specifically from eggs, in Japan. Because the biological effect of dietary lipids on TC is likely to be consistent over time, the timerelated changes observed in these 3 cross-sectional epidemiological cohorts appear to be attributed to this reverse causality. However, the present study cannot confirm this. Results stratified by age group indicated that the aging population during the period examined did not confound the relationships because younger and older age groups showed attenuation between 1980/1990 and 2010 . However, whether time-related changes are related to other historical backgrounds or confounding factors, such as the characteristics of each dataset, warrants consideration. Although the composition of serum cholesterol might have changed, attenuation in the relationship of the Keys score and non-HDL-cholesterol was also observed in a similar fashion.

Keys et al reported that SFA intake strongly correlated with CHD death rates in the seven countries study. ${ }^{24}$ The Keys equation, in which the subduplicate power of dietary cholesterol multiplied by 1.5 , is the most accurate number for predicating TC based on 39 experimental studies. ${ }^{25}$ Other experimental studies confirmed that a $100 \mathrm{mg} /$ day change in dietary cholesterol reduced serum TC concentrations by $2.5 \mathrm{mg} / \mathrm{dL}$ on average. ${ }^{26}$ Because Japanese individuals eat as much as $350-450 \mathrm{mg} /$ day of dietary 
cholesterol (equivalent of 2 eggs), 27 the effects of dietary cholesterol on TC are not negligible considering the population strategy in Japan. Following the abolishment of the upper limit of the recommended dietary cholesterol in the USA in $2015,{ }^{28}$ the USDA added that this abolishment did not suggest that dietary cholesterol was no longer important to consider because the healthy eating pattern in the dietary guidelines limited dietary cholesterol to less than $300 \mathrm{mg} /$ day. $^{29}$

However, recent large-scale observational studies and meta-analyses have demonstrated that a high intake of dietary cholesterol or SFA was related to lower total mortality and CVD or non-CVD mortality. ${ }^{30-32}$ Because humans are animals that can think and act for themselves, it is very natural that participants with high TC or other CVD risk factors would restrict their food intake or select healthy foods according to health information or their doctor's guidance. A previous study conducted in Japan in 1990 reported that SFA intake and the Keys score correlated with TC. ${ }^{33}$ Therefore, a shift in the social background related to the reverse direction between dietary lipids and TC may have occurred between 1990 and 2010. If this hypothesis is accurate, negative relationships between dietary cholesterol and SFA with CVD risk in observational studies need to be interpreted very carefully.

Regarding other factors, BMI was identified as a more important determinant of TC concentration irrespective of sex. Similar to the Keys score, the coefficient values in BMI decreased between 1980/1990 and 2010, which is consistent with previous findings showing that the relationship between being overweight and a high TC concentration gradually weakened between 1980 and 2010.34 Reverse causality may have occurred in BMI, similar to the Keys score; in recent years individuals with higher TC have attempted to decrease their weight more than those with lower TC. In women, age was the most strongly associated factor with TC, which may be related to changes associated with menopause..$^{35}$ The reason why TC gradually increased between 1980 and 2010 in men and women may be the aging of the population and increases in BMI.

In terms of dietary fatty acids not included in the calculation of the Keys score, TFA positively correlated with TC in men and women. MUFA also positively correlated with TC. Another study reported that the effects of TFA on the ratio of low-density lipoprotein (LDL) cholesterol to HDL-cholesterol were significantly stronger than those of SFA. ${ }^{36}$ However, the effects of MUFA on TC remain controversial. ${ }^{37,38}$ Our results suggest that not only dietary cholesterol, SFA, and PUFA, but also TFA and MUFA need to be considered as influential on TC in future studies. Accordingly, the Keys equation would need to be developed according to the dietary and lifestyle factors identified/ assessed after the Keys equation was established. In other words, the addition of BMI, TFA and MUFA into the Keys equation might be possible, and the prediction of LDL-cholesterol instead of TC would be more beneficial according to the new scientific findings to date.

\section{Study Strengths and Limitations}

The present study had several strengths. Our study participants were from the NHNS, which was conducted in 300 randomly selected districts across Japan in 1980, 1990, and 2010. Therefore, our data were representative of Japanese in each era. Additionally, to the best of our knowledge, this is the first study to demonstrate time-related changes in the relationships between dietary lipids and TC in Japanese representative populations. Our study also had some limitations. First, the methods of dietary surveys differed between 1980/1990 and 2010: 1-day household surveys in $1980 / 1990$ and 3-day individual surveys in 2010. Although both were dietary record methods, the difference between $1980 / 1990$ and 2010 possibly had an effect on the results in 2010. Second, because it was an observational study, it was difficult to completely control unknown confounding factors. Third, the response rate of the NHNS has been decreasing, especially for blood tests, ${ }^{\mathbf{8} 39}$ and the small sample size of 2010 has been affected by this. Thus, the generalizability of the dataset in 2010 might be limited compared with the other datasets. Fourth, information on the treatment of hypercholesterolemia was unobtainable for the datasets in 1980. However, we assume that there were few people under treatment for hypercholesterolemia at that time because statins only became a major treatment in Japan from 1989. ${ }^{1}$

\section{Conclusions}

In men and women, a higher Keys score and increased intake of dietary lipids, particularly dietary cholesterol and SFA, correlated with higher TC concentrations in 1980; however, this relationship was attenuated in 1990. A similar relationship was completely absent or further weakened in the dataset of 2010; an inverse correlation was observed in 40-59-year-old men. These results may possibly be related to the recent increase in awareness of hypercholesterolemia and knowledge of dietary improvement in Japan.

\section{Acknowledgments}

\section{The Chairpersons}

Hirotsugu Ueshima (Center for Epidemiologic Research in Asia, Shiga University of Medical Science, Shiga) and Akira Okayama (Research Institute of Strategy for Prevention, Tokyo) for NIPPON DATA80; Hirotsugu Ueshima and Tomonori Okamura (Department of Preventive Medicine and Public Health, Keio University, Tokyo) for NIPPON DATA90; and Katsuyuki Miura (Center for Epidemiologic Research in Asia, Shiga University of Medical Science, Shiga) and Akira Okayama for NIPPON DATA2010.

Research Group Members

Akira Fujiyoshi (Shiga University of Medical Science, Shiga), Atsushi Hozawa (Tohoku Medical Megabank Organization, Tohoku University, Miyagi), Aya Kadota (Shiga University of Medical Science, Shiga), Fumiyoshi Kasagi (Institute of Radiation Epidemiology, Radiation Effects Association, Tokyo), Hideaki Nakagawa (Kanazawa Medical University, Ishikawa), Hideaki Toyoshima (Nagoya University, Aichi), Hiroshi Yanagawa (Jichi Medical University, Tochigi), Katsushi Yoshita (Osaka City University Graduate School of Human Life Science, Osaka), Kazunori Kodama (Radiation Effects Research Foundation, Hiroshima), Kazuo Ueda (Murakami Memorial Hospital, Oita), Kiyomi Sakata (Iwate Medical University, Iwate), Koji Tamakoshi (Nagoya University Graduate School of Medicine, Aichi), Nagako Okuda (University of Human Arts and Sciences, Saitama), Naomi Miyamatsu (Shiga University of Medical Science, Shiga), Nobuo Nishi (National Institutes of Biomedical Innovation, Health and Nutrition, Tokyo), Shigeyuki Saitoh (Sapporo Medical University, Hokkaido), Shinkan Tokudome (Nagoya City University Graduate School of Medical Sciences, Aichi), Takayoshi Okubo (Teikyo University School of Medicine, Tokyo), Takehito Hayakawa [Fukushima Medical University, Fukushima (now at the Kinugasa Research Organization, Ritsumeikan University, Kyoto)], Toru Izumi (Kanazawa Medical University Medical Research Institute, Kitasato University School of Medicine, Kanagawa), Toshiyuki Ojima (Hamamatsu University School of Medicine, Shizuoka), Yasuhiro Matsumura (Bunkyo University, Kanagawa), Yoshihiro Miyamoto (National Cerebral and Cardiovascular Center, Osaka), Yoshikuni Kita (Tsuruga Nursing University, Fukui), Yoshitaka Murakami (Toho University, Tokyo), Yoshikazu Nakamura (Jichi Medical University, Tochigi), and Yutaka Kiyohara (Hisayama 
Research Institute for Lifestyle Diseases, Fukuoka).

\section{Sources of Funding}

This study was supported by a Grant-in-Aid from the Ministry of Health and Welfare under the auspices of the Japanese Association for Cerebro-cardiovascular Diseases Control, the Research Grant for Cardiovascular Diseases (7A-2) from the Ministry of Health, Labour and Welfare, a Health and Labor Sciences Research Grant, Japan (Comprehensive Research on Aging and Health [H11-Chouju-046, H14-Chouju-003, H17-Chouju-012, H19-Chouju-Ippan-014] and Comprehensive Research on Lifestyle Related Diseases including Cardiovascular Diseases and Diabetes Mellitus [H22-JunkankitouSeishuu-Sitei-017, H25-Junkankitou-Seishuu-Sitei-022]), and a Grant-in-Aid from the Japan Society for the Promotion of Science (JSPS) fellows (15J12632).

\section{References}

1. Robinson JG, Stone NJ. The 2013 ACC/AHA guideline on the treatment of blood cholesterol to reduce atherosclerotic cardiovascular disease risk: A new paradigm supported by more evidence. Eur Heart J 2015; 36: 2110-2118.

2. Goto Y, Nakamura H, Hata Y, Goto M, Nakamura Y, Kikuchi A, et al. The meaning of dietary factors in hyperlipidemia: Study from the dietary survey in patients with hyperlipidemia. $J$ Ther 1969; 51: 1551-1557 (in Japanese).

3. Goto Y, Takiyama Y, Shigematsu H, Umetada Y, Ebata Y, Kikuchi A, et al. Cholesterol content in each food. $J$ Ther 1970; 52: 1389-1393 (in Japanese).

4. Okamura T, Sugiyama D, Tanaka T, Dohi S. Worksite wellness for the primary and secondary prevention of cardiovascular disease in Japan: The current delivery system and future directions. Prog Cardiovasc Dis 2014; 56: 515-521.

5. Nakamura Y, Iso H, Kita Y, Ueshima H, Okada K, Konishi M, et al. Egg consumption, serum total cholesterol concentrations and coronary heart disease incidence: Japan Public Health Center-based prospective study. Br J Nutr 2006; 96: 921-928.

6. Shekelle RB, Stamler J, Paul O, Shryock AM, Liu S, Lepper M. Dietary lipids and serum cholesterol level: Change in diet confounds the cross-sectional association. Am J Epidemiol 1982; 115: $506-514$.

7. Okuda N, Miura K, Yoshita K, Matsumura Y, Okayama A, Nakamura $\mathrm{Y}$, et al. Integration of data from NIPPON DATA80/90 and National Nutrition Survey in Japan: For cohort studies of representative Japanese on nutrition. J Epidemiol 2010; 20(Suppl 3): S506-S514.

8. Kadota A, Okuda N, Ohkubo T, Okamura T, Nishi N, Ueshima $\mathrm{H}$, et al. The National Integrated Project for Prospective Observation of Non-communicable Disease And its Trends in the Aged 2010 (NIPPON DATA2010): Objectives, design, and population characteristics. J Epidemiol 2018; 28: S2-S9.

9. Nakamura Y, Okuda N, Turin TC, Fujiyoshi A, Okamura T, Hayakawa $\mathrm{T}$, et al. Comparison of the National Nutritional Survey in Japan estimated individual-based nutritional data and NIPPON DATA80 food frequency questionnaires. $J$ Epidemiol 2010; 20(Suppl 3): S582-S586.

10. Ueshima H, Iida M, Shimamoto T, Konishi M, Tanigaki M, Doi $\mathrm{M}$, et al. Dietary intake and serum total cholesterol level: Their relationship to different lifestyles in several Japanese populations. Circulation 1982; 66: 519-526.

11. Shekelle RB, Shryock AM, Paul O, Lepper M, Stamler J, Liu S, et al. Diet, serum cholesterol, and death from coronary heart disease: The Western Electric study. N Engl J Med 1981; 304: $65-70$.

12. Stamler J, Elliott P, Appel L, Chan Q, Buzzard M, Dennis B, et al. Higher blood pressure in middle-aged American adults with less education-role of multiple dietary factors: The INTERMAP study. J Hum Hypertens 2003; 17: 655-775.

13. Keys A, Parlin RW. Serum cholesterol response to changes in dietary lipids. Am J Clin Nutr 1966; 19: 175-181.

14. Nakamura M, Sato S, Shimamoto T. Improvement in Japanese clinical laboratory measurements of total cholesterol and HDLcholesterol by the US Cholesterol Reference Method Laboratory Network. J Atheroscler Thromb 2003; 10: 145-153.

15. Nakamura M, Kiyama M, Kitamura A, Ishikawa $Y$, Sato $S$, Noda H, et al. Revised system to evaluate measurement of blood chemistry data from the Japanese National Health and Nutrition Survey and Prefectural Health and Nutrition Surveys. J Epidemiol 2013; 23: $28-34$.
16. Kawabata J. Dietary cholesterol and phospholipid. J Hokkaido Soc Food Nutr 1961; 6: 74 (in Japanese).

17. Kuroda K. Changes in health-maintaining and -promoting behavior of the Japanese seen through secondary analysis of 'Hoken eisei-kisochosa' and 'Kokuminseikatsu-kisochosa' by the Ministry of Health and Social Welfare: Healthism in post-war Japan. J Socio-Cultural Res Inst Ryukoku Univ Soc Culture 2004; 6: $307-324$ (in Japanese).

18. Nomura K, Kuroda K. Healthism in postwar Japan: Analysis of best-selling books on health. Jpn Sociol Rev 2005; 55: 449-467 (in Japanese).

19. Takeuchi K. The relationship between undergoing a basic health checkup based on the Health and Medical Services Law for the Aged and medical expenditure covered under the National Health Insurance in Japan. Nihon Eiseigaku Zasshi 2002; 56: 673-681 (in Japanese)

20. Nakamura M, Masui S, Oshima A, Okayama A, Ueshima H. Effects of stage-matched repeated individual counseling on smoking cessation: A randomized controlled trial for the high-risk strategy by lifestyle modification (HISLIM) study. Environ Health Prev Med 2004; 9: 152-160.

21. Schneider RJ, Barengo N, Haapala I, Tavella M. Association between dietary habits, education, serum triglycerides and blood cholesterol among women of Cabildo, Buenos Aires. Medicina (B Aires) 2006; 66: 517-525.

22. Ikeda A, Iso H, Toyoshima H, Fujino Y, Mizoue T, Yoshimura $\mathrm{T}$, et al. The relationships between interest for and participation in health screening and risk of mortality: The Japan Collaborative Cohort Study. Prev Med 2005; 41: 767-771.

23. Goryoda S, Nishi N, Hozawa A, Yoshita K, Arai Y, Kondo K, et al. Differences in lifestyle improvements with the intention to prevent cardiovascular diseases by socioeconomic status in a representative Japanese population: NIPPON DATA2010. $J$ Epidemiol 2018; 28: S35-S39.

24. Keys A, Menotti A, Karvonen MJ, Aravanis C, Blackburn H, Buzina R, et al. The diet and 15-year death rate in the seven countries study. Am J Epidemiol 1986; 124: 903-915.

25. Keys A. Serum cholesterol response to dietary cholesterol. Am J Clin Nutr 1984; 40: 351-359.

26. McNamara DJ. Cholesterol intake and plasma cholesterol: An update. J Am Coll Nutr 1997; 16: 530-534.

27. Ueshima H, Okayama A, Saitoh S, Nakagawa H, Rodriguez B, Sakata K, et al. Differences in cardiovascular disease risk factors between Japanese in Japan and Japanese-Americans in Hawaii: The INTERLIPID study. J Hum Hypertens 2003; 17: 631-639.

28. 2015 Dietary Guidelines Advisory Committee. Scientific Report: Advisory Report to the Secretary of Health and Human Services and the Secretary of Agriculture, February 2015. U.S. Department of Agriculture, Washington D.C.

29. DeSalvo KB, Olson R, Casavale KO. Dietary guidelines for Americans. JAMA 2016; 315: 457-458.

30. Berger S, Raman G, Vishwanathan R, Jacques PF, Johnson EJ. Dietary cholesterol and cardiovascular disease: A systematic review and meta-analysis. Am J Clin Nutr 2015; 102: 276-294.

31. Dehghan M, Mente A, Zhang X, Swaminathan S, Li W, Mohan $\mathrm{V}$, et al. Associations of fats and carbohydrate intake with cardiovascular disease and mortality in 18 countries from five continents (PURE): A prospective cohort study. Lancet 2017; 390: $2050-2062$.

32. Rong Y, Chen L, Zhu T, Song Y, Yu M, Shan Z, et al. Egg consumption and risk of coronary heart disease and stroke: Dose-response meta-analysis of prospective cohort studies. $B M J$ 2013; 346: e8539.

33. Nakamura Y, Okuda N, Turin TC, Fujiyoshi A, Okamura T, Hayakawa T, et al. Fatty acids intakes and serum lipid profiles: NIPPON DATA90 and the national nutrition monitoring. $J$ Epidemiol 2010; 20(Suppl 3): S544-S548.

34. Shibata Y, Ojima T, Nakamura M, Kuwabara K, Miyagawa N, Nakamura Y, et al. An association between overweight, obesity and underweight and high serum total cholesterol level over 30 years among the Japanese elderly: The NIPPON DATA 80, 90 and 2010. J Epidemiol, doi:10.2188/jea.JE20170229.

35. Akahoshi M, Soda M, Nakashima E, Shimaoka K, Seto S, Yano $\mathrm{K}$. Effects of menopause on trends of serum cholesterol, blood pressure, and body mass index. Circulation 1996; 94: 61-66.

36. Ascherio A, Katan MB, Zock PL, Stampfer MJ, Willett WC. Trans fatty acids and coronary heart disease. N Engl J Med 1999; 340: $1994-1998$.

37. Mensink RP, Katan MB. Effect of dietary fatty acids on serum lipids and lipoproteins. A meta-analysis of 27 trials. Arterioscler 
Thromb 1992; 12: 911-919.

38. Mensink RP, Zock PL, Kester AD, Katan MB. Effects of dietary fatty acids and carbohydrates on the ratio of serum total to HDL cholesterol and on serum lipids and apolipoproteins: A metaanalysis of 60 controlled trials. Am J Clin Nutr 2003; 77: 1146 1155.

39. Ikeda N, Takimoto H, Imai S, Miyachi M, Nishi N. Data Resource Profile: The Japan National Health and Nutrition Survey (NHNS). Int J Epidemiol 2015; 44: 1842-1849.

\section{Supplementary Files}

Supplementary File 1

Table S1. Relationships between the Keys score and serum total cholesterol $(\mathrm{mg} / \mathrm{dL})$ among all participants, NIPPON DATA80/90/2010, 1980-2010, Japan

Table S2. Relationships between serum total cholesterol ( $\mathrm{mg} / \mathrm{dL})$ and the Keys score, age, BMI, dietary fiber, smoking status, and drinking status by 1 standard deviation among all participants, NIPPON DATA80/90/2010, 1980-2010, Japan

Figure S1. Participant flow chart.

Figure S2. Scatter plots of serum total cholesterol $(\mathrm{mg} / \mathrm{dL})$ and the Keys score stratified by gender, NIPPON DATA80/90/2010, 1980-2010, Japan.

Please find supplementary file(s);

http://dx.doi.org/10.1253/circj.CJ-18-0628 